\title{
A STOCHASTIC COMPETING-SPECIES MODEL AND ERGODICITY
}

\author{
ZENGJING CHEN,* Shandong University \\ REG KULPERGER, ${ }^{* *}$ The University of Western Ontario
}

\begin{abstract}
We consider a classic competing-species model with the rates changed to include Gaussian white noise. We show that if the noise is not too large, then the stochastic version is ergodic. An explicit relation between the noise and the original competing-species parameters gives a sufficient condition for ergodicity.
\end{abstract}

Keywords: Competing species; stochastic; Itô process; Brownian motion; ergodic

2000 Mathematics Subject Classification: Primary 60H10

Secondary 60G99

\section{Introduction}

Consider the coupled ordinary differential equations

$$
\begin{aligned}
& \mathrm{d} x_{t}=x_{t}\left(\mu-\lambda y_{t}+\gamma x_{t}\right) \mathrm{d} t, \\
& \mathrm{~d} y_{t}=y_{t}\left(-\alpha+\beta x_{t}+\delta y_{t}\right) \mathrm{d} t,
\end{aligned}
$$

where $\mu, \lambda, \alpha$, and $\beta$ are positive constants and $\delta$ and $\gamma$ are real-valued constants. This is a well-known classical model of the population dynamics of two interacting species; see, for example, [13]. The pair $\left(x_{t}, y_{t}\right)$ represents the population sizes of the two species at time $t \geq 0$. Under certain conditions on the parameters, the solution produces a limit cycle, that is, the solution lies on a deterministic contour. When the current population size is $(x, y)$, the rates of population growth are

$$
r_{1}(x, y)=\mu-\lambda y+\gamma x \quad \text { and } \quad r_{2}(x, y)=-\alpha+\beta x+\delta y .
$$

When $\gamma=0$ and $\delta=0$, this model becomes the prey-predator process. When $\gamma<0$ and $\delta<0$, the two species have self-limitations in the sense that, for a species of fixed size, the rate of the other species is negative when its population size is large. This case is the well-known competing-species model. Other combinations of $\gamma$ and $\delta$, e.g. $\gamma>0, \delta<0$ and $\gamma>0, \delta>0$, can be considered but, as indicated in [13], they are not of interest as population dynamics models.

Suppose that

$$
r_{1}\left(x^{*}, y^{*}\right)=\mu-\lambda y^{*}+\gamma x^{*}=0 \quad \text { and } \quad r_{2}\left(x^{*}, y^{*}\right)=-\alpha+\beta x^{*}+\delta y^{*}=0
$$

have the simultaneous solution $\left(x^{*}, y^{*}\right) \in \mathbb{R}_{+}^{2}$, where $\mathbb{R}_{+}=\{x: x>0\}$ is the set of positive real numbers. Then both the prey-predator and competing-species models from (1), with initial

Received 29 April 2003; revision received 9 January 2004.

* Postal address: Department of Mathematics, Shandong University, Jinan, 250100, P. R. China.

** Postal address: Department of Statistical and Actuarial Science, The University of Western Ontario, London, Ontario N6A 5B7, Canada. Email address: kulperger@uwo.ca 
condition $\left(x_{0}, y_{0}\right)=(x, y) \in \mathbb{R}_{+}^{2}$, have nice solutions that are periodic and circle about the fixed point $\left(x^{*}, y^{*}\right)$ of the deterministic equations $r_{1}(x, y)=0$ and $r_{2}(x, y)=0$.

In this note, we consider the case in which the rates (2) are subject to random fluctuations. Specifically, $r_{1}\left(x_{t}, y_{t}\right) \mathrm{d} t$ is replaced by $r_{1}\left(x_{t}, y_{t}\right) \mathrm{d} t+\sigma_{1} \mathrm{~d} W_{t}^{(1)}$, that is, the rate is perturbed by Gaussian white noise. The rate $r_{2}$ is similarly perturbed by an independent Gaussian white noise. Thus, we consider the stochastic competing-species model

$$
\begin{aligned}
& \mathrm{d} x_{t}=x_{t}\left(\mu-\lambda y_{t}+\gamma x_{t}\right) \mathrm{d} t+\sigma_{1} x_{t} \mathrm{~d} W_{t}^{(1)}, \\
& \mathrm{d} y_{t}=y_{t}\left(-\alpha+\beta x_{t}+\delta y_{t}\right) \mathrm{d} t+\sigma_{2} y_{t} \mathrm{~d} W_{t}^{(2)},
\end{aligned}
$$

where $\left\{\left(W_{t}^{(1)}, W_{t}^{(2)}\right): t \geq 0\right\}$ is a standard two-dimensional Brownian motion. The rate parameters are the positive constants $\mu, \lambda, \alpha$, and $\beta$ and the negative constants $\gamma$ and $\delta$. The noise parameters are $\sigma_{1}$ and $\sigma_{2}$.

In this paper, we study the ergodicity properties of (3). We show that if the roots of (2) are in $\mathbb{R}_{+}^{2}$ and the noise parameters $\sigma_{1}$ and $\sigma_{2}$ are sufficiently small, then the solution of (3) is ergodic. The method of proof gives an explicit relation between the noise parameters and the rate parameters that guarantees ergodicity. The proof is based on the methodology of [1]. We modify the results there since [1] gave conditions in terms of radial or Euclidean distances that are not readily applicable to a process on $\mathbb{R}_{+}^{2}$.

In Section 2, we discuss some of the recent literature on competing-species models. In Section 3, we discuss the main result, the construction of the associated stopping times, and the proof. The sufficient condition involved in the main result has a very nice geometric interpretation: if a certain ellipse is a subset of $\mathbb{R}_{+}^{2}$ then the process (3) is ergodic. In Section 4 , we discuss and compare our sufficient condition with those obtained in a recent paper by Rudnicki [14]. In Section 5, we discuss some other extensions based on the methods we use. In Appendix A, we show that the solution of (3) is in $\mathbb{R}_{+}^{2}$ with probability 1; Chessa and Fujita [3] showed a similar result. Our proof easily extends to multiple species as it depends only on showing properties of the hitting time of the boundaries of $\mathbb{R}_{+}^{2}$, via a Markov inequality.

\section{Literature review}

Other authors have considered stochastic systems obtained from the classical population dynamics modelled by (1). Gard and Kannan [6] studied the classical prey-predator model with the rates perturbed by Gaussian white noise. They studied (3) in the case that $\gamma<0$ and $\delta<0$, and showed that, on the set of realizations where it remains bounded for all time, the solution converges to the fixed point $\left(x^{*}, y^{*}\right)$ [6, Theorem 3.1] or converges to the boundary of the bounding set [6, Theorem 3.2]. As mentioned above, Chessa and Fujita [3] studied the prey-predator model and showed that the solution remains in $\mathbb{R}_{+}^{2}$. Renshaw [13] gave a good review of the stochastic competing-species models, but did not include ergodic results. Gard [5] studied the transient behaviour of some population dynamics models, including the classic stochastic model we consider here. In a very interesting related paper (discussed in more detail in Section 4), Rudnicki [14] studied a process similar to the one studied here. The diffusion or variance functions in (3) are $\sigma_{1} x$ and $\sigma_{2} y$.

More recently, there has been work on some variations of the diffusion functions. For example, Mao et al. [12] considered diffusion functions of the form $\sigma_{1} x^{m}$ and looked for conditions such that the solution is defined for all time when $m \geq 2$; that is, such that the solution does not explode in finite time. Manthey et al. [11] considered a random two-species interacting population model living on a bounded space $\mathcal{O} \subset \mathbb{R}^{2}$. They noted that ' $\ldots$ as far 
we know the stability of solutions to stochastic partial differential equations of multi-species population dynamics is not well investigated'. We have found that the ergodic properties of the population dynamics of the type of stochastic systems studied here have not been well investigated. In [15], the transient behaviour of stochastic prey-predator systems with timedependent rate parameter was studied, while in [8] the stochastic prey-predator system (3) with $\delta=\gamma=0$ was studied under the condition that $\sigma_{1}, \sigma_{2} \rightarrow 0$. In the latter paper, it was shown that the solution escapes to infinity. In [10], a stochastic competing-species problem similar to (3), but with $\alpha<0$, was studied, and the probability of extinction obtained.

The proof of our main result is based on the ideas of [1], where conditions were given in terms of radial or Euclidean distances $F(x, y)=\left(x^{2}+y^{2}\right)^{1 / 2}$. This is not a natural distance for us to use, as the solution to (3) takes values in the upper quadrant, that is, in the region $\mathbb{R}_{+}^{2}$. Mao et al. [12] used a distance function

$$
F(x, y)=\sqrt{x}-\log (x)+\sqrt{y}-\log (y),
$$

which enabled them to show that their process is nonexplosive. This function is not well suited to showing ergodicity in the competing-species model. Rather, in this paper, we use the function

$$
F(x, y)=A_{1} x-A_{2} \log (x)+A_{3} y-A_{4} \log (y)+c,
$$

with judicious choices for the constants $A_{1}, A_{2}, A_{3}, A_{4}$, and $c$, to play the role of the contour based on the distance $\left(x^{2}+y^{2}\right)^{1 / 2}$ in [1]. For example, in Theorem 1, we take $A_{1}=\beta, A_{2}=\alpha$, $A_{3}=\lambda, A_{4}=\mu$, and

$$
c=-\alpha+\alpha \log \left(\frac{\alpha}{\beta}\right)-\mu+\mu \log \left(\frac{\mu}{\lambda}\right) .
$$

In particular, with appropriate constants in (4), in Section 4 we are able to weaken the sufficient condition in [14]. Thus, this sufficient condition is not necessary for the process to be ergodic.

The literature on deterministic population dynamics is still quite active. A recent example is [9], in which the prey-predator system with rate parameters having a periodic component was studied. Many of these equations have solutions that behave nicely under conditions in which either the initial condition is perturbed or the rates are perturbed in a deterministic manner. One consequence of the results shown here is that, under a random perturbation of the rates, the process can change from being stable over time to being unstable. In some cases, the process may be ergodic under small random permutations.

\section{Competing species, ergodicity, and ellipses}

Consider the competing-species model (3), where $\mu, \lambda, \alpha$, and $\beta$ are positive constants. In this section we give Theorem 2 , the main result.

In Lemma 1, we rewrite the process in a useful form, which is then studied in Theorem 1. The proof of this theorem is based on finding an appropriate nonnegative function tending to infinity at the boundaries of $\mathbb{R}_{+}^{2}$ and judicious use of bounds on expectations of some stopping times. This leads to a nice geometric condition sufficient for ergodicity.

Lemma 1. Suppose that $\gamma \delta>0$; that is, either $\gamma<0$ and $\delta<0$ or $\gamma>0$ and $\delta>0$. Let $\varepsilon_{1}^{\prime}=\gamma / \beta$ and $\varepsilon_{2}^{\prime}=\delta / \lambda$. Rewrite (3) as

$$
\begin{aligned}
& \mathrm{d} x_{t}=x_{t}\left[\mu^{\prime}-\lambda y_{t}+\varepsilon_{1}^{\prime}\left(\beta x_{t}-\alpha^{\prime}\right)\right] \mathrm{d} t+\sigma_{1} x_{t} \mathrm{~d} W_{t}^{(1)}, \\
& \mathrm{d} y_{t}=y_{t}\left[-\alpha^{\prime}+\beta x_{t}+\varepsilon_{2}^{\prime}\left(\lambda y_{t}-\mu^{\prime}\right)\right] \mathrm{d} t+\sigma_{2} y_{t} \mathrm{~d} W_{t}^{(2)},
\end{aligned}
$$


for appropriate constants $\mu^{\prime}$ and $\alpha^{\prime}$. If either

(i) $\gamma>0$ and $0<\delta<\alpha \lambda / \mu$, or

(ii) $\delta<0$ and $-\mu \beta / \alpha<\gamma<0$,

then $\mu^{\prime}>0$ and $\alpha^{\prime}>0$.

Proof. Comparing the coupled stochastic differential equation (SDE) in this lemma with (3) yields

$$
\varepsilon_{1}^{\prime}=\frac{\gamma}{\beta}, \quad \varepsilon_{2}^{\prime}=\frac{\delta}{\lambda}, \quad \mu^{\prime}-\varepsilon_{1}^{\prime} \alpha^{\prime}=\mu, \quad \alpha^{\prime}+\varepsilon_{2}^{\prime} \mu^{\prime}=\alpha .
$$

Solving for $\mu^{\prime}$ and $\alpha^{\prime}$ gives

$$
\mu^{\prime}=\frac{\mu+\varepsilon_{1}^{\prime} \alpha}{1+\varepsilon_{1}^{\prime} \varepsilon_{2}^{\prime}}, \quad \alpha^{\prime}=\frac{\alpha-\varepsilon_{2}^{\prime} \mu}{1+\varepsilon_{1}^{\prime} \varepsilon_{2}^{\prime}} .
$$

When $\varepsilon_{1}^{\prime} \varepsilon_{2}^{\prime}>0$, we have $\mu^{\prime}>0$ and $\alpha^{\prime}>0$ if and only if either $\gamma>0$ and $0<\delta<\alpha \lambda / \mu$ or $\delta<0$ and $-\mu \beta / \alpha<\gamma<0$, as required. This completes the proof of Lemma 1.

The form of the SDE in Lemma 1 is of use in proving ergodicity, whereas the original form (3) is not so immediately useful.

Theorem 1. Consider the SDE process

$$
\begin{aligned}
& \mathrm{d} x_{t}=x_{t}\left[\mu-\lambda y_{t}+\varepsilon_{1}\left(\beta x_{t}-\alpha\right)\right] \mathrm{d} t+\sigma_{1} x_{t} \mathrm{~d} W_{t}^{(1)}, \\
& \mathrm{d} y_{t}=y_{t}\left[-\alpha+\beta x_{t}+\varepsilon_{2}\left(\lambda y_{t}-\mu\right)\right] \mathrm{d} t+\sigma_{2} y_{t} \mathrm{~d} W_{t}^{(2)},
\end{aligned}
$$

where $\mu, \lambda, \alpha$, and $\beta$ are positive constants, with initial condition $\left(x_{0}, y_{0}\right)=(x, y) \in \mathbb{R}_{+}^{2}$. If $\varepsilon_{2}<0$ and $\varepsilon_{1}<0$ are such that

$$
0<\frac{\sigma_{1}^{2} \alpha+\sigma_{2}^{2} \mu}{2 \alpha^{2}}<-\varepsilon_{1} \text { and } 0<\frac{\sigma_{1}^{2} \alpha+\sigma_{2}^{2} \mu}{2 \mu^{2}}<-\varepsilon_{2},
$$

then the solution to the SDE (5) is ergodic.

Theorem 2. Consider the stochastic competing-species process (3). Suppose that $\gamma<0$ and $\delta<0$ and let $\varepsilon_{1}^{\prime}=\gamma / \beta$ and $\varepsilon_{2}^{\prime}=\delta / \lambda$. Also suppose that

$$
0<\frac{\sigma_{1}^{2} \alpha^{\prime}+\sigma_{2}^{2} \mu^{\prime}}{2\left(\alpha^{\prime}\right)^{2}}<-\varepsilon_{1}^{\prime}=-\frac{\gamma}{\beta}<\frac{\mu}{\alpha} \quad \text { and } \quad 0<\frac{\sigma_{1}^{2} \alpha^{\prime}+\sigma_{2}^{2} \mu^{\prime}}{2\left(\mu^{\prime}\right)^{2}}<-\varepsilon_{2}^{\prime},
$$

where

$$
\mu^{\prime}=\frac{\mu+\varepsilon_{1}^{\prime} \alpha}{1+\varepsilon_{1}^{\prime} \varepsilon_{2}^{\prime}} \quad \text { and } \quad \alpha^{\prime}=\frac{\alpha-\varepsilon_{2}^{\prime} \mu}{1+\varepsilon_{1}^{\prime} \varepsilon_{2}^{\prime}} .
$$

Then the solution to (3) is ergodic.

Proof. Theorem 2 is a corollary of Theorem 1, which applies since, by Lemma 1, we have $\alpha^{\prime}>0$ and $\mu^{\prime}>0$. 
Remark 1. Theorem 2 says that if the noise coefficients $\sigma_{1}$ and $\sigma_{2}$ are sufficiently small, if the damping terms $\gamma x_{t}$ and $\delta y_{t}$ in the drift of the $x$ component and $y$ component in (3), respectively, are negative, and if one further condition holds, then the stochastic competing-species process is ergodic. The extra condition is $-\beta \mu / \alpha<\gamma<0$. The damping term $\delta y_{t}$ in the drift of the $y$ component only requires $\delta<0$ for the system to be ergodic.

Notice that the solution to (2) with vanishing rates is

$$
\left(\begin{array}{c}
x^{*} \\
y^{*}
\end{array}\right)=\frac{1}{\gamma \delta+\beta \lambda}\left(\begin{array}{c}
\lambda \alpha-\delta \mu \\
\beta \mu+\gamma \alpha
\end{array}\right)
$$

Thus, $x^{*}>0$ but $y^{*}>0$ if and only if $\beta \mu+\gamma \alpha>0$ or, equivalently, if and only if $\mu / \alpha>-\gamma / \beta$. This extra condition is very natural, requiring that the deterministic competing-species system has a fixed point in $\mathbb{R}_{+}^{2}$. Hence, if the self-damping effect of $x_{t}$ is not too large, if the noise is sufficiently small, and if the deterministic system has a fixed point in $\mathbb{R}_{+}^{2}$, then the stochastic competing-species model is ergodic.

The proof of Theorem 1 can be modified to show that if the noise is sufficiently large, the stochastic competing-species process is transient. This is discussed at the end of this section.

Proof of Theorem 1. Let

$$
F(x, y)=\beta x-\alpha \log (x)+\lambda y-\mu \log (y)+c,
$$

where

$$
c=-\alpha+\alpha \log \left(\frac{\alpha}{\beta}\right)-\mu+\mu \log \left(\frac{\mu}{\lambda}\right) .
$$

Thus $F(\alpha / \beta, \mu / \lambda)=0$ and $F(x, y)>0$ if $(x, y) \neq(\alpha / \beta, \mu / \lambda)$. Also, $F(x, y) \rightarrow \infty$ as $(x, y)$ tends to the boundary of $\mathbb{R}_{+}^{2}$. This choice of $F$ is a particular case of (4) with $A_{1}=\beta$, $A_{2}=\alpha, A_{3}=\lambda$, and $A_{4}=\mu$.

An application of Itô's lemma to $F_{t}=F\left(x_{t}, y_{t}\right)$ yields

$$
\begin{aligned}
\mathrm{d} F_{t}= & {\left[\varepsilon_{1}\left(\beta x_{t}-\alpha\right)^{2}+\varepsilon_{2}\left(\lambda y_{t}-\mu\right)^{2}+\frac{1}{2}\left(\sigma_{1}^{2} \alpha+\sigma_{2}^{2} \mu\right)\right] \mathrm{d} t } \\
& +\sigma_{1}\left(\beta x_{t}-\alpha\right) \mathrm{d} W_{t}^{(1)}+\sigma_{2}\left(\lambda y_{t}-\mu\right) \mathrm{d} W_{t}^{(2)} .
\end{aligned}
$$

Since $\varepsilon_{1}<0$ and $\varepsilon_{2}<0$, the drift is bounded above in $\mathbb{R}_{+}^{2}$ and, thus, by Lemma 3 , below, the solution is in $\mathbb{R}_{+}^{2}$ with probability 1 . Consider the region

$$
D=\left\{(x, y): \frac{(x-\alpha / \beta)^{2}}{\left(\sigma_{1}^{2} \alpha+\sigma_{2}^{2} \mu\right) / 2\left|\varepsilon_{1}\right| \beta^{2}}+\frac{(y-\mu / \lambda)^{2}}{\left(\sigma_{1}^{2} \alpha+\sigma_{2}^{2} \mu\right) / 2\left|\varepsilon_{2}\right| \lambda^{2}} \leq 1\right\},
$$

which has an ellipse as its boundary. Notice that

$$
\begin{aligned}
& \left|\varepsilon_{1}\right|>\frac{\sigma_{1}^{2} \alpha+\sigma_{2}^{2} \mu}{2 \alpha^{2}} \Rightarrow \frac{\alpha}{\beta}>\sqrt{\frac{\sigma_{1}^{2} \alpha+\sigma_{2}^{2} \mu}{2\left|\varepsilon_{1}\right| \beta^{2}}}, \\
& \left|\varepsilon_{2}\right|>\frac{\sigma_{1}^{2} \alpha+\sigma_{2}^{2} \mu}{2 \mu^{2}} \Rightarrow \frac{\mu}{\lambda}>\sqrt{\frac{\sigma_{1}^{2} \alpha+\sigma_{2}^{2} \mu}{2\left|\varepsilon_{2}\right| \lambda^{2}}} .
\end{aligned}
$$

Thus, the conditions on $\varepsilon_{1}$ and $\varepsilon_{2}$ imply that $D \subset \mathbb{R}_{+}^{2}$. 
Let $k=\frac{1}{2}\left(\sigma_{1}^{2} \alpha+\sigma_{2}^{2} \mu\right)$. There then exists a constant $d>0$ such that

$$
\frac{\alpha}{\beta}-\left(\sqrt{\frac{k}{\left|\varepsilon_{1}\right| \beta^{2}}}+d\right)>0 \text { and } \frac{\mu}{\lambda}-\left(\sqrt{\frac{k}{\left|\varepsilon_{2}\right| \lambda^{2}}}+d\right)>0,
$$

which imply that

$$
D^{*}=\left\{(x, y): \frac{(x-\alpha / \beta)^{2}}{\left(\sqrt{k /\left|\varepsilon_{1}\right| \beta^{2}}+d\right)^{2}}+\frac{(y-\mu / \lambda)^{2}}{\left(\sqrt{k /\left|\varepsilon_{2}\right| \lambda^{2}}+d\right)^{2}} \leq 1\right\} \subset \mathbb{R}_{+}^{2} .
$$

To be specific, we can take

$$
d=\frac{1}{2}\left(\frac{\alpha}{\beta}-\sqrt{\frac{\sigma_{1}^{2} \alpha+\sigma_{2}^{2} \mu}{2\left|\varepsilon_{1}\right| \beta^{2}}}\right) \wedge\left(\frac{\mu}{\lambda}-\sqrt{\frac{\sigma_{1}^{2} \alpha+\sigma_{2}^{2} \mu}{2\left|\varepsilon_{2}\right| \lambda^{2}}}\right) .
$$

Since $d>0$, we also have $D \subset D^{*} \subset \mathbb{R}_{+}^{2}$.

Consider $D^{* \mathrm{c}}$ relative to $\mathbb{R}_{+}^{2}$ :

$$
\begin{aligned}
D^{* \mathrm{c}} & =\left\{(x, y) \in \mathbb{R}_{+}^{2}: \frac{(x-\alpha / \beta)^{2}}{\left(\sqrt{k /\left|\varepsilon_{1}\right| \beta^{2}}+d\right)^{2}}+\frac{(y-\mu / \lambda)^{2}}{\left(\sqrt{k /\left|\varepsilon_{2}\right| \lambda^{2}}+d\right)^{2}}>1\right\} \\
& =\left\{(x, y) \in \mathbb{R}_{+}^{2}: \frac{\left|\varepsilon_{1}\right|(\beta x-\alpha)^{2}}{\left(\sqrt{k}+\sqrt{\left|\varepsilon_{1}\right|} \beta d\right)^{2}}+\frac{\left|\varepsilon_{2}\right|(\lambda y-\mu)^{2}}{\left(\sqrt{k}+\sqrt{\left|\varepsilon_{2}\right|} \lambda d\right)^{2}}>1\right\} .
\end{aligned}
$$

Therefore, if $(x, y) \in D^{* \mathrm{c}}$ then

$$
\left|\varepsilon_{1}\right|(\beta x-\alpha)^{2}+\left|\varepsilon_{2}\right|(\lambda y-\mu)^{2}>\left(\sqrt{k}+\min \left\{\sqrt{\left|\varepsilon_{1}\right|} \beta d, \sqrt{\left|\varepsilon_{2}\right|} \lambda d\right\}\right)^{2} \geq k+d_{0},
$$

where

$$
d_{0}:=\min \left\{\sqrt{\left|\varepsilon_{1}\right|} \beta d, \sqrt{\left|\varepsilon_{2}\right|} \lambda d\right\}^{2}>0 .
$$

It follows that, for $(x, y) \notin D^{*}$ and $(x, y) \in \mathbb{R}_{+}^{2}$,

$$
-\left|\varepsilon_{1}\right|(\beta x-\alpha)^{2}-\left|\varepsilon_{2}\right|(\lambda y-\mu)^{2}+\frac{1}{2}\left(\sigma_{1}^{2} \alpha+\sigma_{2}^{2} \mu\right) \leq-d_{0}<0
$$

or, since $\varepsilon_{1}, \varepsilon_{2}<0$, that

$$
\varepsilon_{1}(\beta x-\alpha)^{2}+\varepsilon_{2}(\lambda y-\mu)^{2}+\frac{1}{2}\left(\sigma_{1}^{2} \alpha+\sigma_{2}^{2} \mu\right) \leq-d_{0}<0 .
$$

Let $(x, y) \in \mathbb{R}_{+}^{2}-D^{*}$, and let $\left(x_{t}, y_{t}\right)$ be the solution to (5) with initial value $(x, y)$. Let $\tau=\inf \left\{t \geq 0:\left(x_{t}, y_{t}\right) \in D^{*}\right\}$, let $m$ be sufficiently large that

$$
D^{*} \subset D_{m}:=\left(\frac{1}{m}, m\right) \times\left(\frac{1}{m}, m\right),
$$

and define $\tau_{m}=\inf \left\{t \geq 0:\left(x_{t}, y_{t}\right) \notin D_{m}\right\}$. By Lemma 3, $\tau_{m} \rightarrow \infty$ almost surely as $m \rightarrow \infty$.

Below, $\mathrm{E}_{(x, y)}$ and $\mathrm{P}_{(x, y)}$ respectively denote the expectation and probability measure for the diffusion process with initial condition $\left(x_{0}, y_{0}\right)=(x, y)$. From (7), we have

$$
\mathrm{E}_{(x, y)} F\left(x_{\tau \wedge \tau_{m}}, y_{\tau \wedge \tau_{m}}\right) \leq F(x, y)-d_{0} \mathrm{E}_{(x, y)}\left[\tau \wedge \tau_{m}\right]
$$




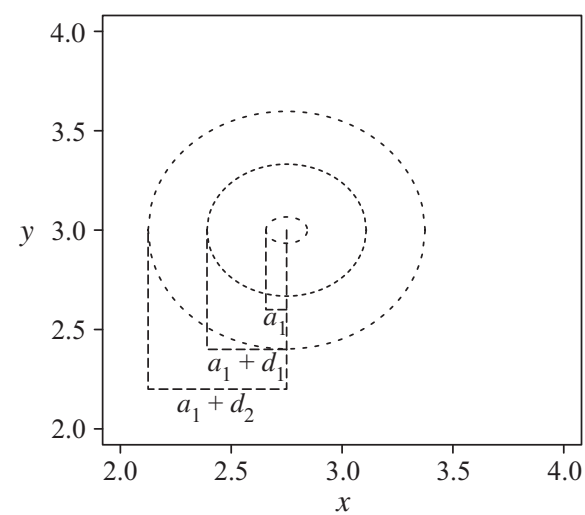

FIGURE 1: Nested ellipses and their semimajor axes.

Since $F(x, y)>0$ for $(x, y) \neq(\alpha / \beta, \mu / \lambda)$, it follows that

$$
\begin{aligned}
\mathrm{E}_{(x, y)} F\left(x_{\tau \wedge \tau_{m}}, y_{\tau \wedge \tau_{m}}\right) & \geq \mathrm{E}_{(x, y)}\left[F\left(x_{\tau_{m}}, y_{\tau_{m}}\right) \mathbf{1}\left(\tau \geq \tau_{m}\right)\right] \\
& \geq \min _{(u, v) \in \partial D_{m}} F(u, v) \mathrm{P}_{(x, y)}\left[\tau \geq \tau_{m}\right],
\end{aligned}
$$

where $\mathbf{1}(\cdot)$ denotes the indicator function. Clearly,

$$
\min _{(u, v) \in \partial D_{m}} F(u, v) \rightarrow \infty \quad \text { as } m \rightarrow \infty
$$

and, thus,

$$
\mathrm{P}_{(x, y)}\left[\tau \geq \tau_{m}\right] \leq \frac{F(x, y)}{\min _{(u, v) \in \partial D_{m}} F(u, v)} \rightarrow 0
$$

as $m \rightarrow \infty$. Since $\tau_{m} \rightarrow \infty$, we have $\mathrm{P}_{(x, y)}[\tau=\infty]=0$ and, hence, $\left(x_{t}, y_{t}\right)$ is recurrent.

From (7), we also have

$$
\mathrm{E}_{(x, y)}\left[\tau \wedge \tau_{m}\right] \leq \frac{1}{d_{0}} F(x, y) .
$$

By an application of the monotone convergence theorem and the convergence $\tau_{m} \rightarrow \infty$, we thus have

$$
\mathrm{E}_{(x, y)} \tau \leq \frac{1}{d_{0}} F(x, y) .
$$

Furthermore we can choose $a_{1}>0$ and $b_{1}>0$ such that the ellipse

$$
D^{* *}=\left\{(x, y): \frac{(x-\alpha / \beta)^{2}}{a_{1}^{2}}+\frac{(y-\mu / \lambda)^{2}}{b_{1}^{2}}=1\right\}
$$

lies between the boundaries of $D^{*}$ and $D_{m}$.

Figure 1 shows nested ellipses illustrating the construction of $D, D^{*}$, and $D^{* *}$. The figure shows the semimajor axes (which lie in the $x$-direction). The semimajor axis of the central ellipse has length $a_{1}$, while those of the other two ellipses have lengths $a_{1}+d_{1}$ and $a_{1}+d_{2}$. Since, by the choice of $a_{1}, \alpha / \beta-a_{1}>0$, it is clear that we can choose $d_{1}$ and $d_{2}$ to satisfy $0<d_{1}<d_{2}$ and still have the ellipses $D^{*}$ and $D^{* *}$ to the right of a line $x=1 / m$, where 
$1 / m<\alpha / \beta-a_{1}$. Similarly, we can simultaneously choose $d_{1}$ and $d_{2}$, with $0<d_{1}<d_{2}$, so as to have the three nested ellipses in the rectangular region $D_{m}$. Figure 1 uses the competing-species parameters

$$
\left(\frac{\alpha \lambda-\gamma \mu}{\gamma \delta+\beta \lambda}, \frac{\alpha \delta+\beta \mu}{\gamma \delta+\beta \lambda}\right)=\left(\frac{8}{3}, \frac{1}{6}\right) .
$$

Since $D^{* *} \subset D^{* \mathrm{c}}$, we have

$$
\sup _{(x, y) \in D^{* *}} \mathrm{E}_{(x, y)} \tau \leq \frac{1}{d_{0}} \sup _{(x, y) \in D^{* *}} F(x, y)<\infty .
$$

Notice that an ellipse centred at $(\alpha / \beta, \mu / \lambda)$ can be viewed as measuring the distance from $(x, y)$ to $(\alpha / \beta, \mu / \lambda)$. In the construction above, we have three nested ellipsoidal regions

$$
D \subset D^{*} \subset \text { convex hull }\left(D^{* *}\right) .
$$

$D^{*}$ is open and contains $(\alpha / \beta, \mu / \lambda)$ in its interior. Applying [1, Lemma 3.4] then shows that the process (5) is ergodic.

This concludes the proof of Theorem 1 .

Remark 2. We should note that [1, Lemma 3.4], which is based on the natural Euclidean distance in $\mathbb{R}^{2}$, uses circles. However, said lemma follows from [1, Lemma 2.6], which applies to open sets $U$ and their closures $\bar{U}$. By choosing these to be ellipses, it is immediate that [1, Lemma 3.4] can be restated in terms of nested ellipses. We now give the result corresponding to [1, Lemma 3.4]. To satisfy the conditions of this lemma, we let $D^{*}$ correspond to the open 'ball' of radius $r_{0}>0$ around $z=(\alpha / \beta, \mu / \lambda)$, and let $D^{* *}$ correspond to the contour at distance $r_{1}>r_{0}$.

Theorem 3. Suppose that one of the following is true:

(i) $\gamma<0, \delta>0$, and $\left(2 \mu-\sigma_{1}^{2}\right) /\left(2 \alpha+\sigma_{2}^{2}\right)<\min \{-\gamma / \beta, \lambda / \delta\}$;

(ii) $\gamma<0, \delta<0$, and $\left(2 \mu-\sigma_{1}^{2}\right) /\left(2 \alpha+\sigma_{2}^{2}\right)<-\gamma / \beta$;

(iii) $\gamma>0$ and $\delta>0$.

Then the process (5) is transient.

Proof. We first prove that the claim follows from part (i). Choose two positive constants, $A_{2}$ and $A_{4}$, such that $A_{4} / A_{2}=\min \{-\gamma / \beta, \lambda / \delta\}$. Then

$$
\gamma A_{2}+\beta A_{4} \leq 0, \quad \delta A_{4}-\lambda A_{2} \leq 0 .
$$

In (4), let $A_{1}=A_{3}=0$ and $c=0$. Writing $F_{t}=F\left(x_{t}, y_{t}\right)$, and applying Itô's formula, we have

$$
\begin{aligned}
-\mathrm{d} F_{t}= & {\left[A_{2}\left(\mu-\frac{1}{2} \sigma_{1}^{2}\right)-A_{4}\left(\alpha+\frac{1}{2} \sigma_{2}^{2}\right)+\left(\delta A_{4}-\lambda A_{2}\right) y_{t}+\left(\gamma A_{2}+\beta A_{4}\right) x_{t}\right] \mathrm{d} t } \\
& +A_{2} \sigma_{1} \mathrm{~d} W_{t}^{(1)}+A_{4} \sigma_{2} \mathrm{~d} W_{t}^{(2)} \\
\leq & {\left[A_{2}\left(\mu-\frac{1}{2} \sigma_{1}^{2}\right)-A_{4}\left(\alpha+\frac{1}{2} \sigma_{2}^{2}\right)\right] \mathrm{d} t+A_{2} \sigma_{1} \mathrm{~d} W_{t}^{(1)}+A_{4} \sigma_{2} \mathrm{~d} W_{t}^{(2)} . }
\end{aligned}
$$

Since $A_{2}\left(\mu-\frac{1}{2} \sigma_{1}^{2}\right)-A_{4}\left(\alpha+\frac{1}{2} \sigma_{2}^{2}\right)<0$ and

$$
\limsup _{t \rightarrow \infty} \frac{\left|W_{t}^{(i)}\right|}{\sqrt{2 t \log \log t}}=1, \quad i=1,2,
$$


we have

$$
\left[A_{2}\left(\mu-\frac{1}{2} \sigma_{1}^{2}\right)-A_{4}\left(\alpha+\frac{1}{2} \sigma_{2}^{2}\right)\right] t+A_{2} \sigma_{1} W_{t}^{(1)}+A_{4} \sigma_{2} W_{t}^{(2)} \rightarrow-\infty .
$$

Hence,

$$
-F\left(x_{t}, y_{t}\right) \rightarrow-\infty, \quad t \rightarrow \infty
$$

and, since

$$
-F\left(x_{t}, y_{t}\right)=\log \left(x_{t}^{A_{2}}\right) \log \left(y_{t}^{A_{4}}\right)=\log \left(x_{t}^{A_{2}} y_{t}^{A_{4}}\right)
$$

and the constants $A_{2}$ and $A_{4}$ are positive, we obtain $x_{t}^{A_{2}} y_{t}^{A_{4}} \rightarrow 0$. Thus, $\left(x_{t}, y_{t}\right)$ is transient.

The proof that the claim follows from part (ii) is similar to the previous argument, and is actually the result of [14].

We now prove that the claim follows from part (iii). Take an $\ell>0$ such that $\frac{1}{2} \ell \sigma_{1}^{2}<\varepsilon_{1}$ and $\frac{1}{2} \ell \sigma_{2}^{2}<\varepsilon_{2}$, and define

$$
H(x)=\int_{1}^{x} \mathrm{e}^{-\ell t} \mathrm{~d} t .
$$

Then $\lim _{x \rightarrow \infty} H(x)<\infty$. Recall the definition of $F$ in (6). From (7), we have

$$
\begin{aligned}
\mathrm{d} H\left(F_{t}\right)= & H^{\prime}\left(F_{t}\right)\left[\varepsilon_{1}\left(\beta x_{t}-\alpha\right)^{2}+\varepsilon_{2}\left(\lambda y_{t}-\mu\right)^{2}+\frac{1}{2}\left(\sigma_{1}^{2} \alpha+\sigma_{2}^{2} \mu\right)\right] \mathrm{d} t \\
& +\frac{1}{2} H^{\prime \prime}\left(F_{t}\right)\left[\sigma_{1}^{2}\left(\beta x_{t}-\alpha\right)^{2}+\sigma_{2}^{2}\left(\lambda y_{t}-\mu\right)^{2}\right] \mathrm{d} t \\
& +H^{\prime}\left(F_{t}\right) \sigma_{1}\left(\beta x_{t}-\alpha\right) \mathrm{d} W_{t}^{(1)}+H^{\prime}\left(F_{t}\right) \sigma_{2}\left(\lambda y_{t}-\mu\right) \mathrm{d} W_{t}^{(2)} \\
= & \mathrm{e}^{-\ell F_{t}}\left[\left(\varepsilon_{1}-\frac{1}{2} \ell \sigma_{1}^{2}\right)\left(\beta x_{t}-\alpha\right)^{2}+\left(\varepsilon_{2}-\frac{1}{2} \ell \sigma_{2}^{2}\right)\left(\lambda y_{t}-\mu\right)^{2}+\frac{1}{2}\left(\sigma_{1}^{2} \alpha+\sigma_{2}^{2} \mu\right)\right] \mathrm{d} t \\
& +\mathrm{e}^{-\ell F_{t}} \sigma_{1}\left(\beta x_{t}-\alpha\right) \mathrm{d} W_{t}^{(1)}+\mathrm{e}^{-\ell F_{t}} \sigma_{2}\left(\lambda y_{t}-\mu\right) \mathrm{d} W_{t}^{(2)} .
\end{aligned}
$$

Let $D(r)=\{(x, y): F(x, y) \leq r\}$. Fix an $r_{0}>0$ and consider the region $D\left(r_{0}\right)$. Suppose that $(x, y) \notin D\left(r_{0}\right)$ and let $\tau$ be the hitting time of the set $D\left(r_{0}\right)$ for the process (5) with starting value $(x, y)$. We will show that $\mathrm{P}_{(x, y)}(\tau<\infty)<1$ or, equivalently, $\mathrm{P}_{(x, y)}(\tau=\infty)>0$.

There exists an $r>r_{0}$ such that $(x, y)$ is in the interior of $D(r)$. Let

$$
\tau_{r}=\inf \left\{t \geq 0:\left(x_{t}, y_{t}\right) \in D(r)\right\}
$$

be the hitting time of $D(r)$. From Lemma 3 and the properties of $F$, it follows that $\tau_{r} \rightarrow \infty$ as $r \rightarrow \infty$. Taking expectations, we then have

$$
\begin{aligned}
\mathrm{E}_{(x, y)} H\left(F_{\tau \wedge \tau_{r}}\right)= & H(F(x, y)) \\
& +\mathrm{E}_{(x, y)}\left[\int _ { 0 } ^ { \tau \wedge \tau _ { r } } \mathrm { e } ^ { - \ell F _ { t } } \left[\left(\varepsilon_{1}-\frac{1}{2} \ell \sigma_{1}^{2}\right)\left(\beta x_{t}-\alpha\right)^{2}\right.\right. \\
& \left.\left.\quad+\left(\varepsilon_{2}-\frac{1}{2} \ell \sigma_{2}^{2}\right)\left(\lambda y_{t}-\mu\right)^{2}+\frac{1}{2}\left(\sigma_{1}^{2} \alpha+\sigma_{2}^{2} \mu\right)\right] \mathrm{d} t\right] \\
\geq & H(F(x, y)),
\end{aligned}
$$

since the integrand is nonnegative. Note that $H\left(F_{\tau}\right)=H\left(r_{0}\right)$ and $H\left(F_{\tau_{r}}\right)=H(r)$; thus

$$
\begin{aligned}
H(F(x, y)) & \leq \mathrm{E}_{(x, y)} H\left(F_{\tau \wedge \tau_{r}}\right) \\
& =\mathrm{E}_{(x, y)}\left[H\left(F_{\tau}\right) \mathbf{1}\left(\tau \leq \tau_{r}\right)\right]+\mathrm{E}\left[H\left(F_{\tau_{r}}\right) \mathbf{1}\left(\tau>\tau_{r}\right)\right] \\
& =H\left(r_{0}\right) \mathrm{P}_{(x, y)}\left[\tau \leq \tau_{r}\right]+H(r) \mathrm{P}_{(x, y)}\left[\tau>\tau_{r}\right] \\
& =H\left(r_{0}\right)+\left(H(r)-H\left(r_{0}\right)\right) \mathrm{P}_{(x, y)}\left[\tau>\tau_{r}\right]
\end{aligned}
$$


and, therefore,

$$
\mathrm{P}_{(x, y)}\left[\tau>\tau_{r}\right] \geq \frac{H(F(x, y))-H\left(r_{0}\right)}{H(r)-H\left(r_{0}\right)} .
$$

By letting $r \rightarrow \infty$, we obtain

$$
\mathrm{P}_{(x, y)}[\tau=\infty] \geq \frac{H(F(x, y))-H\left(r_{0}\right)}{H(r)-H\left(r_{0}\right)}>0
$$

This concludes the proof of Theorem 3 .

\section{Comparison with other results}

In this section, we will make a detailed comparison of our results with those of [14]. In order to facilitate this comparison, in this section we use the notation of [14]. Table 1 gives the correspondence between parameters.

Rudnicki [14] studied a system related to ours, but with the Brownian noise $W^{(1)}=W^{(2)}$. He also made some statements on the case in which $W^{(1)}$ and $W^{(2)}$ are independent Brownian motions. His main results concern a system that has a singular diffusion noise term. We study a case in which $W^{(1)}$ and $W^{(2)}$ are independent Brownian motions. Our method is based on the idea of [1] and thus requires a nonsingular diffusion. Hence, we make comparisons only with the relevant parts of [14].

Rudnicki [14, Lemma 2] constructed the solution to an SDE as the solution of an operator; see his equations (19)-(21). This is equivalent to obtaining a strong solution to the SDE (3); that is, the solution is a specific functional of the Brownian motions $W^{(1)}$ and $W^{(2)}$. This is a very interesting idea, which may be exploited further. Rudnicki [14, Lemma 5] then gave the proof of the main part of his Theorem 1, based on a Khasminskii function.

We have the analogue of [14, Theorem 1(III)] in our Theorem 3, and [14, Theorem 1(I)] is a sufficient condition slightly different from our Theorem 2. We will show in Lemma 2, below, that if $\delta c_{1}-\mu c_{2}=0$ then (3) is ergodic under certain conditions on $\sigma$ and $\rho$. Thus, while $\delta c_{1}-\mu c_{2}>0$ is sufficient, it is not necessary.

Our proof is based on exploiting a function of the form (4). When $A_{i}>0, i=1,2,3,4$, we may take

$$
c=A_{2}\left(\log \left(\frac{A_{2}}{A_{1}}\right)-1\right)+A_{4}\left(\log \left(\frac{A_{4}}{A_{3}}\right)-1\right)
$$

TABLE 1: The correspondence between our notation and that of Rudnicki [14].

\begin{tabular}{cc}
\hline This paper & Rudnicki [14] \\
\hline$\mu$ & $\alpha$ \\
$\lambda$ & $\beta$ \\
$-\gamma$ & $\mu$ \\
$\alpha$ & $\gamma$ \\
$\beta$ & $\delta$ \\
$-\delta$ & $v$ \\
$\sigma_{1}$ & $\sigma$ \\
$\sigma_{2}$ & $\rho$ \\
\hline
\end{tabular}


so that $F>0$ except at $(x, y)=\left(A_{2} / A_{1}, A_{4} / A_{3}\right)$ (at which point $F=0$ ), and $F(x, y) \rightarrow \infty$ as $(x, y)$ tends to the boundary of $\mathbb{R}_{+}^{2}$. From Itô's lemma applied to (3), we then obtain

$$
\mathrm{d} F_{t}=H\left(x_{t}, y_{t}\right) \mathrm{d} t+\mathrm{d} M_{t},
$$

where $M_{t}$ is a zero-mean martingale and $H(x, y)$ is a quadratic function of $x$ and $y$.

Theorem 4. Suppose that we can find positive constants $A_{1}, A_{2}, A_{3}$, and $A_{4}$ for the function (4) such that $H(x, y)=0$ is an ellipsoidal subset of $\mathbb{R}_{+}^{2}$, where $H$ is the drift function above. Equivalent conditions on $H$ are that

(i) $H(x, y)>0$ on the interior of this ellipse, and

(ii) $H(x, y)<0$ on the exterior of this ellipse, which is guaranteed if

$$
H(0,0)<0, \quad H(x, 0)<0, \quad H(0, y)<0 .
$$

Then the SDE (3) is ergodic.

Proof. We can proceed, in the manner of the proof of Theorem 1, to construct bounds on the stopping times of return to the central region and, hence, obtain ergodicity using the method of [1]. This concludes the proof.

Rudnicki [14, Theorem 1(I)] obtained a result of asymptotic stability under the sufficient conditions $c_{1}>0$ and $\delta c_{1}-\mu c_{2}>0$, where $c_{1}=\alpha-\frac{1}{2} \sigma^{2}$ and $c_{2}=\gamma+\frac{1}{2} \rho^{2}$.

Choose constants $A_{1}=1, A_{2}=k_{1}, A_{3}=A$, and $A_{4}=A k_{2}$, whence

$$
F(x, y)=x-k_{1} \log (x)+A\left(y-k_{2} \log (y)\right)+c .
$$

Then

$$
\begin{aligned}
H(x, y)= & -\mu\left(x-k_{1}\right)^{2}-A v\left(y-k_{2}\right)^{2}+(-\beta+A \delta)\left(x-k_{1}\right)\left(y-k_{2}\right) \\
& +\left(\alpha-\mu k_{1}-\beta k_{2}\right)\left(x-k_{1}\right)-A\left(\gamma-\delta k_{1}+v k_{2}\right)\left(y-k_{2}\right) \\
& +\frac{1}{2}\left(\sigma^{2} k_{1}+A \rho^{2} k_{2}\right) .
\end{aligned}
$$

After some algebra, we obtain

$$
H(0,0)=-k_{1}\left(\alpha-\frac{1}{2} \sigma^{2}\right)+A k_{2}\left(\gamma+\frac{1}{2} \rho^{2}\right)=-k_{1} c_{1}+A k_{2} c_{2} .
$$

The contour $H(x, y)=0$ is an ellipse if and only if

$$
(A \delta-\beta)^{2}-4 A \mu \nu<0,
$$

and the ellipse is a subset of $\mathbb{R}_{+}^{2}$ if $H(x, 0)<0$ and $H(0, y)<0$. The function $H(x, 0)$ is a quadratic form in $x$ and, hence, $H(x, 0)<0$ for all $x$ if and only if $H(x, 0)=0$ has no real roots, since the coefficient of $x$ is negative. Similarly, $H(0, y)<0$ if and only if $H(0, y)=0$ has no real roots. Thus, a set of conditions equivalent to those guaranteeing condition (ii) of Theorem 4 is

$$
\begin{aligned}
4 \mu\left(-c_{1} k_{1}+A c_{2} k_{2}\right)+\left(\alpha+\mu k_{1}-A \delta k_{2}\right)^{2} & <0, \\
4 A \nu\left(-c_{1} k_{1}+A c_{2} k_{2}\right)+\left(-A \gamma+\beta k_{1}+A \nu k_{2}\right)^{2} & <0, \\
(-\beta+A \delta)^{2}-4 A \mu \nu & <0 .
\end{aligned}
$$


Finally, we take $k_{1}=b \delta$ and $A k_{2}=b \mu$, for some positive constant $b$. Then $H(0,0)=$ $-b\left(\delta c_{1}-\mu c_{2}\right)$ and the conditions of Theorem 4 are satisfied if $\delta c_{1}-\mu c_{2}>0, b>0$, $\alpha^{2}<4 \mu b\left(\delta c_{1}-\mu c_{2}\right)$, and

$$
[b(\mu \nu+\beta \delta)-A \gamma]^{2}<4 A \nu b\left(\delta c_{1}-\mu c_{2}\right) .
$$

Since $A$ and $b$ are arbitrary, we can take

$$
A=b \frac{(\mu \nu+\beta \delta)}{\gamma}
$$

such that the previous inequality holds. Thus, we obtain the analogue of [14, Theorem 1(I)], that is, the ergodicity of (3).

The condition $\left(\delta c_{1}-\mu c_{2}\right)>0$ can be weakened to include $\left(\delta c_{1}-\mu c_{2}\right)=0$. It is also assumed that the deterministic competing-species differential equation has a fixed point in $\mathbb{R}_{+}^{2}$.

Lemma 2. Suppose that $\left(\delta c_{1}-\mu c_{2}\right)=0$. Then the system of equations

$$
\begin{aligned}
\alpha-\mu k_{1}-\beta k_{2} & =0, \\
\gamma-\delta k_{1}+\nu k_{2} & =0,
\end{aligned}
$$

has a positive solution $\left(k_{1}, k_{2}\right)$. Let $A=\beta / \delta$.

If $\sigma>0$ and $\rho>0$ satisfy the following inequalities then (3) is ergodic:

$$
\frac{1}{2}\left(\sigma^{2} k_{1}+\rho^{2} A k_{2}\right)<\mu k_{1}^{2}, \quad \frac{1}{2}\left(\sigma^{2} k_{1}+\rho^{2} A k_{2}\right)<A \nu k_{2} .
$$

Proof. The proof follows from the method used in the proof of Theorem 1, once we have chosen the positive constants $k_{1}, k_{2}$, and $A$, in (8), such that the ellipse $H(x, y)=0$ is a subset of $\mathbb{R}_{+}^{2}$. Then the stopping times constructed from this ellipse are obtained, and Battacharya's results will apply as they did to Theorem 1 .

In (8), we take $k_{1}$ and $k_{2}$ to satisfy

$$
\begin{gathered}
\alpha-\mu k_{1}-\beta k_{2}=0, \\
\gamma-\delta k_{1}+\nu k_{2}=0,
\end{gathered}
$$

that is,

$$
k_{1}=\frac{\beta \gamma+\alpha \nu}{\beta \delta+\mu \nu}, \quad k_{2}=\frac{\alpha \delta-\gamma \mu}{\beta \delta+\mu \nu} .
$$

Clearly, $k_{1}>0$ and $k_{2}>0$ if and only if $\alpha \delta-\gamma \mu>0$. Note that

$$
\begin{aligned}
\alpha \delta-\gamma \mu & =\delta\left(\alpha-\frac{1}{2} \sigma^{2}+\frac{1}{2} \sigma^{2}\right)-\mu\left(\gamma+\frac{1}{2} \rho^{2}-\frac{1}{2} \rho^{2}\right) \\
& =\delta c_{1}-\mu c_{2}+\frac{1}{2}\left(\delta \sigma^{2}+\mu \rho^{2}\right) \\
& =\frac{1}{2}\left(\delta \sigma^{2}+\mu \rho^{2}\right)
\end{aligned}
$$

is positive, since $\delta c_{1}-\mu c_{2}=0$. Now $H$ simplifies to

$$
\begin{aligned}
H(x, y)= & -\mu\left(x-k_{1}\right)^{2}-A v\left(y-k_{2}\right)^{2}+(-\beta+A \delta)\left(x-k_{1}\right)\left(y-k_{2}\right) \\
& +\frac{1}{2}\left(\sigma^{2} k_{1}+A \rho^{2} k_{2}\right) .
\end{aligned}
$$


Since $A=\beta / \delta$, we see that $H$ simplifies further to

$$
H(x, y)=-\mu\left(x-k_{1}\right)^{2}-A v\left(y-k_{2}\right)^{2}+\frac{1}{2}\left(\sigma^{2} k_{1}+A \rho^{2} k_{2}\right) .
$$

Notice that the constants $k_{1}, k_{2}$, and $A$ are all positive and do not depend on the noise coefficients $\sigma$ and $\rho$.

Next consider the ellipse $H(x, y)=0$, the set of pairs $(x, y)$ satisfying

$$
\mu\left(x-k_{1}\right)^{2}+A v\left(y-k_{2}\right)^{2}=\frac{1}{2}\left(\sigma^{2} k_{1}+A \rho^{2} k_{2}\right)=: \ell
$$

or, equivalently,

$$
\frac{\left(x-k_{1}\right)^{2}}{\ell / \mu}+\frac{\left(y-k_{2}\right)^{2}}{\ell / A \nu}=1 .
$$

This will be a subset of $\mathbb{R}_{+}^{2}$ when the conditions of the lemma hold.

This concludes the proof of Lemma 2.

\section{Discussion}

We have shown that, under certain conditions and bounds on the noise, the stochastic competing-species process (3) is ergodic. The method of proof involves a very natural geometric object, an ellipse. The region $D$, given below (7) in the proof, plays a key role. If it is contained in the interior of $\mathbb{R}_{+}^{2}$ then the process is ergodic.

A special case of (3) is when the extra damping terms with coefficients $\gamma$ and $\delta$ are zero, that is, $\gamma=\delta=0$. This is the prey-predator system. In this case, (7) becomes

$$
\mathrm{d} F_{t}=\frac{1}{2}\left(\sigma_{1}^{2} \alpha+\sigma_{2}^{2} \mu\right) \mathrm{d} t+\sigma_{1}\left(\beta x_{t}-\alpha\right) \mathrm{d} W_{t}^{(1)}+\sigma_{2}\left(\lambda y_{t}-\mu\right) \mathrm{d} W_{t}^{(2)}
$$

and, hence, has constant positive drift when the noise coefficients are positive. There is no ability to control this drift in this case. The stochastic prey-predator system, given by (3) with $\gamma=\delta=0$, is not ergodic if there is any noise present. In fact, we find that

$$
\mathrm{E} F\left(x_{t}, y_{t}\right)=\frac{1}{2}\left(\sigma_{1}^{2} \alpha+\sigma_{2}^{2} \mu\right) t+F\left(x_{0}, y_{0}\right) \rightarrow \infty
$$

as $t \rightarrow \infty$. Since $F(x, y) \rightarrow \infty$ as $(x, y)$ tends to the boundary of $\mathbb{R}_{+}^{2}$, the pair $\left(x_{t}, y_{t}\right)$ tends in probability to the boundary of $\mathbb{R}_{+}^{2}$ as $t \rightarrow \infty$. However, this result does not say to where on the boundary $\left(x_{t}, y_{t}\right)$ goes: it could tend to $(0,0)$ or $x_{t}$ could tend to infinity. A classic paper [4] discussed a class of diffusions that wander out to infinity, although this stochastic prey-predator system does not obey the conditions in that paper. The result for the stochastic prey-predator system is very different from the stable case of the classical deterministic one, that is (3) with no noise. This problem was further studied in [2].

The stochastic prey-predator process is also quite different from the stochastic competingspecies process (3). With any noise parameter $\sigma_{1}>0$ or $\sigma_{2}>0$ it is never ergodic, whereas (3) is ergodic with sufficiently small but nonzero noise.

We could interpret a stochastic version of (3) as a Stratonovich integral (S-integral) instead of an Itô integral. We then have

$$
\begin{aligned}
& \mathrm{d} x_{t}=x_{t}\left(\mu-\lambda y_{t}+\gamma x_{t}\right) \mathrm{d} t \pm \sigma_{1} x_{t} \circ \mathrm{d} W_{t}^{(1)}, \\
& \mathrm{d} y_{t}=y_{t}\left(-\alpha+\beta x_{t}+\delta y_{t}\right) \mathrm{d} t \pm \sigma_{2} y_{t} \circ \mathrm{d} W_{t}^{(2)},
\end{aligned}
$$


where $b\left(x_{t}\right) \circ \mathrm{d} W_{t}$ denotes the Stratonovich integral. We consider the cases of adding or subtracting the $\mathrm{S}$-integral separately, as they lead to different results. The $\mathrm{S}$-integral is related to the Itô integral by

$$
b\left(x_{t}\right) \circ \mathrm{d} W_{t}=\frac{1}{2} \frac{\mathrm{d} b\left(x_{t}\right)}{\mathrm{d} x} b\left(x_{t}\right) \mathrm{d} W_{t} .
$$

If the diffusion coefficient function is $b(x)=\sigma x$, then

$$
b\left(x_{t}\right) \circ \mathrm{d} W_{t}=\frac{1}{2} \sigma^{2} x_{t}+\sigma x_{t} \mathrm{~d} W_{t} .
$$

Thus, (9) gives the equivalent Itô process

$$
\begin{aligned}
& \mathrm{d} x_{t}=x_{t}\left(\left[\mu \pm \frac{1}{2} \sigma_{1}^{2}\right]-\lambda y_{t}+\gamma x_{t}\right) \mathrm{d} t+\sigma_{1} x_{t} \mathrm{~d} W_{t}^{(1)}, \\
& \mathrm{d} y_{t}=y_{t}\left(-\left[\alpha \mp \frac{1}{2} \sigma_{2}^{2}\right]+\beta x_{t}+\delta y_{t}\right) \mathrm{d} t+\sigma_{2} y_{t} \mathrm{~d} W_{t}^{(2)} .
\end{aligned}
$$

This is of the form (3), with the added constraints

$$
\mu \pm \frac{1}{2} \sigma_{1}^{2}>0 \text { and } \alpha \mp \frac{1}{2} \sigma_{2}^{2}>0 .
$$

Theorem 1 will again yield a result of the form of Theorem 2, provided that the above positivity conditions hold. The weakest version of these will be for $\sigma_{1}>0$ and $\sigma_{2}<0$. If the noise parameters $\sigma_{1}$ and $\sigma_{2}$ are sufficiently small then the S-integral stochastic competing-species process (9) is ergodic. If either of the noise parameters is too large then the ergodicity conditions will fail. Karlin and Taylor [7, p. 352] made some remarks about the use of Itô integrals versus Stratonovich integrals in applications of stochastic integrals.

In Lemma 2, we are also able to weaken the condition of Rudnicki [14] for the ergodicity of (3) to $\left(\delta c_{1}-\mu c_{2}\right) \geq 0$. Rudnicki has a slightly different conclusion - that the process is asymptotically stable - while our conclusion is that the process is ergodic.

\section{Appendix A.}

This section gives two technical lemmas, for completeness. The first says that the stochastic competing-species solution is defined for all finite time and, in particular, that the solution does not reach the boundary of $\mathbb{R}_{+}^{2}$ in finite time. The second says that, for any compact set, if the process starts from the interior it will hit the boundary in finite expected time.

Chessa and Fujita [3] proved that a stochastic prey-predator process of the type studied here has solution on $\mathbb{R}_{+}^{2}$. Below, we show that this holds more generally as the proof is based on an appropriate choice of nonnegative function used to show that the boundary is not reached in finite time.

Lemma 3. Let $\left(x_{t}, y_{t}\right)$ be an Itô process on $\mathbb{R}_{+}^{2}$ with respect to the two-dimensional standard Brownian motion $W_{t}=\left(W_{t}^{(1)}, W_{t}^{(2)}\right)^{\top}$ (where $\boldsymbol{u}^{\top}$ means transpose of the matrix $\boldsymbol{u}$ ), with initial value $(x, y) \in \mathbb{R}_{+}^{2}$. Suppose that there exists a positive function $F: \mathbb{R}_{+}^{2} \rightarrow \mathbb{R}_{+}$, such that $F(x, y) \rightarrow \infty$ as $(x, y)$ converges to the boundary of $\mathbb{R}_{+}^{2}$. Suppose also that

$$
\mathrm{d} F\left(x_{t}, y_{t}\right)=f\left(x_{t}, y_{t}\right) \mathrm{d} t+\sigma\left(x_{t}, y_{t}\right) \mathrm{d} W_{t},
$$

where $\sigma(\cdot, \cdot)$ is continuous and $f(x, y) \leq c$ for some constant $c$. Let

$$
\tau=\inf \left\{t \geq 0:\left(x_{t}, y_{t}\right) \in \partial \mathbb{R}_{+}^{2}\right\}
$$

be the hitting time of reaching the boundary $\partial \mathbb{R}_{+}^{2}$ of $\mathbb{R}_{+}^{2}$. Then $\mathrm{P}[\tau=\infty]=1$. 
Proof. Let $m>0$ be an integer such that

$$
(x, y) \in K_{m}:=\left(\frac{1}{m}, m\right) \times\left(\frac{1}{m}, m\right),
$$

and define

$$
\tau_{m}=\inf \left\{t \geq 0:\left(x_{t}, y_{t}\right) \notin K_{m}\right\} .
$$

Then $\tau \geq \tau_{m}$ and

$$
\mathrm{E} F\left(x_{\tau_{m} \wedge t}, y_{\tau_{m} \wedge t}\right) \leq F(x, y)+c t .
$$

Since $\min _{(u, v) \in \partial K_{m}} F(u, v) \rightarrow \infty$ as $m \rightarrow \infty$, we have

$$
\mathrm{P}\left[\tau_{m}<t\right] \leq \frac{F(x, y)+c t}{\min _{(u, v) \in \partial K_{m}} F(u, v)} \rightarrow 0 \quad \text { as } m \rightarrow \infty .
$$

Since $\mathrm{P}[\tau<t] \leq \mathrm{P}\left[\tau_{m}<t\right]$ for all $m>0$ sufficiently large, it follows that $\mathrm{P}[\tau<t]=0$ for all finite $t$. This concludes the proof of Lemma 3 .

Lemma 4. Let $D$ be any closed subset of $\mathbb{R}_{+}^{2}$. Consider the SDE (5) with initial condition $\left(x_{0}, y_{0}\right) \in D^{o}$, the interior of $D$. Let

$$
\tau=\inf \left\{t \geq 0:\left(x_{t}, y_{t}\right) \in \partial D\right\}
$$

be the hitting time of the boundary of $D$. Then $\mathrm{E}_{\left(x_{0}, y_{0}\right)} \tau<\infty$.

Proof. Consider the functions $F$, given by (6), and $H(u)=\mathrm{e}^{c u}$, where $c$ is a positive constant to be determined later. Notice that $H$ is nonnegative and monotone increasing. Let $F_{t}=F\left(x_{t}, y_{t}\right)$ and $a=\frac{1}{2}\left(\sigma_{1}^{2} \alpha+\sigma_{2}^{2} \mu\right)$. Then

$$
\mathrm{d} H\left(F_{t}\right)=L\left(x_{t}, y_{t}\right) \mathrm{d} t+\mathrm{d} M_{t},
$$

where $M_{t}$ is a zero-mean martingale and the drift term is given by the function

$$
\begin{aligned}
L(x, y)= & c H(F(x, y))(a-\{\mid \\
& \left.\varepsilon_{1}\left|(\beta x-\alpha)^{2}+\right| \varepsilon_{2} \mid(\lambda y-\mu)^{2}\right\} \\
& \left.+\frac{1}{2} c\left\{\sigma_{1}^{2}(\beta x-\alpha)^{2}+\sigma_{2}^{2}(\lambda y-\mu)^{2}\right\}\right) \\
= & c H(F(x, y))\left(a+\left\{\left(\frac{1}{2} c \sigma_{1}^{2}-\left|\varepsilon_{1}\right|\right)(\beta x-\alpha)^{2}+\left(\frac{1}{2} c \sigma_{2}^{2}-\left|\varepsilon_{2}\right|\right)(\lambda y-\mu)^{2}\right\}\right) .
\end{aligned}
$$

Since $D$ is bounded and $L$ is continuous, we can choose a $c$ such that $\eta=\inf _{(x, y) \in D} L(x, y)>0$ :

$$
c>\max \left\{\frac{2\left|\varepsilon_{1}\right|}{\sigma_{1}^{2}}, \frac{2\left|\varepsilon_{2}\right|}{\sigma_{2}^{2}}\right\} .
$$

Then

$$
\begin{aligned}
\mathrm{E}_{\left(x_{0}, y_{0}\right)} H\left(F_{\tau}\right) & =H\left(F_{0}\right)+\mathrm{E}_{\left(x_{0}, y_{0}\right)} \int_{0}^{\tau} L\left(x_{t}, y_{t}\right) \mathrm{d} t \\
& \geq H\left(F_{0}\right)+\eta \mathrm{E}_{\left(x_{0}, y_{0}\right)} \tau
\end{aligned}
$$

and, thus,

$$
\sup _{(x, y) \in \bar{D}} H(F(x, y)) \geq \mathrm{E}_{\left(x_{0}, y_{0}\right)} H\left(F_{\tau}\right) \geq H\left(F_{0}\right)+\eta \mathrm{E}_{\left(x_{0}, y_{0}\right)} \tau,
$$

where $\bar{D}$ is the closure of $D$, and

$$
\mathrm{E}_{\left(x_{0}, y_{0}\right)} \tau \leq \frac{1}{\eta} \sup _{(x, y) \in \bar{D}} H(F(x, y))<\infty .
$$

This concludes the proof of Lemma 4. 


\section{Acknowledgements}

The authors thank the referee for detailed comments and pointing out the recently accepted and now published paper [14]. The referee's remarks led us to a deeper understanding of the role of the contour level function and its role in obtaining ergodic results for certain diffusions on $\mathbb{R}_{+}^{2}$.

This work was done while Zengjing Chen was visiting The University of Western Ontario in 2003. He deeply appreciates their hospitality, as well as funding from the Fields Institute. This work was also supported by grants from the Natural Sciences and Engineering Research Council of Canada.

\section{References}

[1] Bhattacharya, R. N. (1978). Criteria for recurrence and existence of invariant measures for multidimensional diffusions. Ann. Prob. 6, 541-553.

[2] Chen, Z. And Kulperger, R. (2003). A stochastic prey predator process and damping. In preparation.

[3] Chessa, S. and Fujita Y. H. (2002). The stochastic equation of predator-prey population dynamics. Boll. Unione Mat. Ital. Sez. B. Artic. Ric. Mat. 5, 789-804 (in Italian).

[4] Friedman, A. (1973). Wandering out to infinity of diffusion processes. Trans. Am. Math. Soc. 184, 185-203.

[5] Gard, T. C. (2000). Transient effects of stochastic multi-population models. In Electron. J. Differential Equat., Conf. 05 (Proc. Conf. Nonlinear Differential Equations, Coral Gables, FL, 1999), eds S. Cantrell and C. Cosner, Texas State University, pp. 81-90.

[6] Gard, T. and Kanna, D. (1976). On a stochastic differential equation modeling of prey-predator evolution. J. Appl. Prob. 13, 429-443.

[7] Karlin, S. and Taylor, H. (1981). A Second Course in Stochastic Processes. Academic Press, New York.

[8] Khasminskit, R. Z. And Klebaner, F. C. (2001). Long term behavior of solutions of the Lotka-Volterra system under small random perturbations. Ann. Appl. Prob. 11, 952-963.

[9] KING, A. et al. (1996). Weakly dissipative predator-prey systems. Bull. Math. Biology 58, 835-859.

[10] Mangel, M. and Ludwig, D. (1977). Probability of extinction in a stochastic competition. SIAM J. Appl. Math. 33, 256-266.

[11] Manthey, R. and Maslowski, B. (2002). A random continuous model for two interacting populations. Appl. Math. Optimization 45, 213-236.

[12] Mao, X., Marion, G. and Renshaw, E. (2002). Environmental Brownian noise suppresses explosions in population dynamics. Stoch. Process. Appl. 97, 95-110.

[13] Renshaw, E. (1991). Modelling Biological Populations in Space and Time. Cambridge University Press.

[14] Rudnicki, R. (2003). Long-time behaviour of a stochastic prey-predator model. Stoch. Process. Appl. 108, 93-107.

[15] Spagnola, B. and La Barbera, A. (2002). Role of the noise on the transient dynamics of an ecosystem of interacting species. Physica A 315, 114-124. 